\title{
Body Mass Index, Waist Circumference and Cut-Off Points for Metabolic Syndrome in Urban Residents in Ningxia
}

\author{
Jianjun Yang*, Hongyan Qiu, Hongyu Li, Yuhong Zhang, Xiujuan Tao, Yanna Fan \\ Public Health School, Ningxia Medical University, Yinchuan, China \\ Email: "Yangjianjun_1969@163.com
}

Received 3 October 2015; accepted 5 December 2015; published 8 December 2015

Copyright (C) 2015 by authors and Scientific Research Publishing Inc.

This work is licensed under the Creative Commons Attribution International License (CC BY). http://creativecommons.org/licenses/by/4.0/

(c) (i) Open Access

\section{Abstract}

A growing number of studies show that different countries and populations require different cut-off points for body mass index (BMI), and waist circumference (WC) leading to obesity. There are no data on optimal cut-off points to metabolic syndrome (MS) among urban residents in Ningxia. Our aim is to determine the appropriate cut-off points for BMI and WC associated with elevated prevalent MS risk among urban residents in Ningxia. A total of 2500 urban residents in Ningxia were examined from May 2008 to March 2009 in a community-based cross-sectional study. Height, body weight, waist circumference and hip circumference were measured to calculate BMI. Fasting blood glucose (FBG), plasma levels of triglyceride (TG), total cholesterol (TC), and highdensity lipoprotein cholesterol (HDL-C) were examined using a blood glucose meter and the chromatographic enzyme method. BMI and WC were measured to assess overweight or obesity. Of these, 301 subjects were chosen according to metabolic syndrome diagnosis standards to form the case group. The control group comprised 301 healthy people without diabetes, hypertension, hyperlipemia, coronary heart disease, cerebrovascular disease, malignant tumors or chronic infections. Logistic regression and receiver-operating characteristic (ROC) curve analyses were used to determine optimal cut-off points for BMI and WC in relation to the area under the curve (AUC), sensitivity and specificity. The optimal cut-off points for male and female respectively were 24.78 $\mathrm{kg} / \mathrm{m}^{2}$ (sensitivity $=0.879$, specificity $\left.=0.648\right)$ and $24.72 \mathrm{~kg} / \mathrm{m}^{2}($ sensitivity $=0.804$, specificity $=$ 0.767 ) for BMI, $85.95 \mathrm{~cm}$ (sensitivity $=0.701$, specificity $=0.641$ ) and $78.25 \mathrm{~cm}$ (sensitivity $=0.804$, specificity $=0.528$ ) for WC. Urban residents in Ningxia were at high risk of MS, and the cut-off points for BMI and WC were lower than the data currently recommended in Asian population.

\section{Keywords}

MS, BMI, WC, ROC Curves

${ }^{*}$ Corresponding author.

How to cite this paper: Yang, J.J., Qiu, H.Y., Li, H.Y., Zhang, Y.H., Tao, X.J. and Fan, Y.N. (2015) Body Mass Index, Waist Circumference and Cut-Off Points for Metabolic Syndrome in Urban Residents in Ningxia. Open Journal of Endocrine and Metabolic Diseases, 5, 163-170. http://dx.doi.org/10.4236/ojemd.2015.512020 


\section{Introduction}

With the development of economy and improvement in the living standards, the aging of populations, the spectrum of diseases and the causes of death are increasing. Metabolic syndrome (MS) is a condition that promotes atherosclerosis and increases the risk of cardiovascular disease and diabetes mellitus [1]-[3]. The core of MS is insulin resistance. Obesity and being overweight are important factors causing insulin resistance [4]. The World Health Organization (WHO) recommends the use of pre-specified cut-off points for BMI and WC to standardize comparisons within and between populations [5]. WHO states that a body mass index (BMI) of 25 - 29.9 is overweight; a BMI of $\geq 30$ is obese, and a waist circumference (WC) of $\geq 94 \mathrm{~cm}$ for males and $\geq 80 \mathrm{~cm}$ for females is obese [6]. Currently such cut-off points are derived from studies among European populations and thus may not be applicable to other ethnic groups [7] [8]. A growing number of studies show that different countries and populations require different cut-off points as indicators for detecting MS [9]-[11]. For example, some studies suggest that Asian populations manifest MS risk factors at lower levels of BMI and WC than Western populations, owing in part to a higher percentage of body fat [12] [13].

The Ningxia Hui Autonomous Region is located in the upper reaches of the Yellow River, western China. Because of lack of information on indicators and cut-off points when screening for metabolic syndrome in city dwellers, China is unable to apply these measures to people living in urban and undeveloped area such as Ningxia province. To provide a scientific basis for the screening and prevention of metabolic syndrome in Ningxia we investigated the indicators for detecting metabolic syndrome in Ningxia city residents, and determined appropriate cut-off points.

\section{Materials and Methods}

\subsection{Study Population}

The study was for a chronic disease and the nutrition survey was carried out in the province of Ningxia, China. Data was collected from May 2008 to March 2009 and stratified cluster sampling was used. First, five regions (Shizuishan city, Yingchuan city, Zhongwei city, Qingtongxia city and Guyuan city) with different levels of economic income in Ningxia were selected, and then one community was randomly selected in each region. The sampling database was based on the age-ethnic distribution for Ningxia province (Census 2005). The target group was all composed of Ningxia natives aged $\geq 25$ years who resided in Ningxia for at least four generations. In total, 2500 people were enrolled in the study. The ethics committee of Ningxia Medical University approved all study protocols, and all subjects provided written informed consent.

All participants were interviewed by trained people with a standard closed question survey. Subjects did not report chronic viral infection, cold or flu, acute respiratory infection, dental problems, or any type of surgery in the week preceding the study. Following the International Diabetes Federation (IDF 2005), a participant was defined as having metabolic syndrome if obese (waist circumference $\geq 90 \mathrm{~cm}$ for Chinese men or $\geq 80 \mathrm{~cm}$ for Chinese women) and satisfied at least two of the following: 1) triglyceride (TG) level of $\geq 1.7$ mmol/L; 2) reduced High-density lipoprotein (HDL-C) levels of $<1.03 \mathrm{mmol} / \mathrm{L}$ in men, and $<1.29 \mathrm{mmol} / \mathrm{L}$ in women; 3 ) raised systolic or diastolic blood pressure of 130/85 mmHg or higher, or previously diagnosed hypertension; and 4) a raised fasting plasma glucose (FBG) level of $5.6 \mathrm{mmol} / \mathrm{L}$ or higher, or previously diagnosed type 2 diabetes mellitus. From our sample, 301 people were defined as having metabolic syndrome according to the International Diabetes Federation standard. With gender, age, and area by 1:1 matched, 301 healthy people without diabetes, hypertension, hyperlipemia, coronary heart disease, cerebrovascular disease, malignant tumors or chronic infections were selected as the control group. Meanwhile they must abide by the inclusion and exclusion criteria, and informed consent. The two groups were similar in age and other characteristics.

\subsection{Anthropometric and Biochemistry Characterization}

Standing height was measured once using a portable ruler. Body weight was measured once using scales. BMI was calculated as $\mathrm{kg} / \mathrm{m}^{2}$. Obesity was defined as a BMI $\geq 30$ according to WHO standard guidelines [5]. Arterial blood pressure was measured three times in the sitting position. All participants were at rest for at least 30 min before measurements. Patients with average blood pressure $(\geq 140 / 90 \mathrm{mmHg})$ or taking antihypertensive medication were classified as hypertensive. Blood samples were collected from the antecubital vein between 06:0008:00 h, after $10 \mathrm{~h}$ of fasting and no alcohol. Two sets of fasting blood samples were collected from each subject 
in sodium fluoride potassium oxalate tubes (for glucose) and lithium heparin vacuum tubes (for lipids). Samples were centrifuged at the survey site, and plasma was transferred to separate tubes and labeled and transferred immediately in cold boxes filled with ice $\left(2^{\circ} \mathrm{C}-8^{\circ} \mathrm{C}\right)$. Samples were frozen at $-20^{\circ} \mathrm{C}$ for further analysis. Fast blood sugar, HDL-C and TG levels were measured using the chromatographic enzyme method (OLYMPUS Automatic Biochemical Analyzer, AU400). The intra and inter-assay coefficient of variation for cholesterol and triglycerides did not exceed $4 \%$. Hypercholesterolemia was defined as total serum cholesterol levels $\geq 5.72$ $\mathrm{mmol} / \mathrm{L}$. Blood glucose levels were measured immediately after on-site collection with a Blood Glucose Meter (Life Scan, Milpitas, USA).

\subsection{Data Analysis}

All questionnaires were proofread comprehensively prior to data entry. Illogical data and missing data were verified and supplied. All data were input into EpiData and then exported into SPSS (version 13.0 SPSS Corp, College Station, NX) used for all statistical analysis. Continuous variables were presented as mean values \pm standard deviation and categorical variables are presented as absolute and relative frequencies or prevalence. Indicators for metabolic syndrome were compared using $t$-tests and demographic characters were compared using chi-square tests and $t$-tests. Multivariate logistic regression analysis was applied to control influencing factors and the screening threshold of indicators was determined by receiver operating characteristic (ROC) curves. All reported $\mathrm{p}$ values are based on two-sided tests with a significance level of $5 \%$.

\section{Results}

\subsection{Sample Characteristics}

Six hundred and two adults were studied. In the case group, 301 cases were diagnosed according to the diagnostic criteria for metabolic syndrome. There were no significant differences in age among adults in both groups (Table 1). Except for education and smoking, there were no significant difference for gender, nationality, and female drinking in both groups.

\subsection{Measurement of Indicators for Metabolic Syndrome in Both Groups}

The subjects had higher overall mean BMI, WC, SBP, DBP, FBS, TC, TG, and LDL-C levels in case group, whereas the subjects had significantly higher mean HDL-C in control group (all p < 0.05) (Table 2).

Table 1. Characteristics of case and control groups.

\begin{tabular}{|c|c|c|c|c|c|}
\hline & Characters & Case group & Control group & $t / \chi^{2}$ & $\mathrm{p}$ \\
\hline Age & & $55.02 \pm 0.82$ & $49.95 \pm 0.78$ & -1.815 & $>0.05$ \\
\hline \multirow{2}{*}{ Gender } & Male & 157 & 142 & \multirow{2}{*}{1.599} & \multirow{2}{*}{0.206} \\
\hline & Female & 144 & 159 & & \\
\hline \multirow{3}{*}{ Nationality } & Han & 251 & 264 & \multirow{3}{*}{2.185} & \multirow{3}{*}{0.335} \\
\hline & Hui & 39 & 28 & & \\
\hline & Other & 10 & 9 & & \\
\hline \multirow{3}{*}{ Education } & Primary and below & 151 & 106 & \multirow{3}{*}{13.886} & \multirow{3}{*}{0.01} \\
\hline & Junior middle school & 95 & 130 & & \\
\hline & High school and above & 54 & 64 & & \\
\hline \multirow{4}{*}{ Smoking } & Never & 177 & 166 & \multirow{4}{*}{22.563} & \multirow{4}{*}{0.00} \\
\hline & Sometime & 59 & 102 & & \\
\hline & Every day & 49 & 28 & & \\
\hline & Quitted smoking & 15 & 5 & & \\
\hline \multirow{2}{*}{ Drinking } & Yes & 66 & 68 & \multirow{2}{*}{0.017} & \multirow{2}{*}{0.896} \\
\hline & No & 232 & 233 & & \\
\hline
\end{tabular}




\subsection{Multivariate Logistic Regression Analysis of Metabolic Syndrome}

We assigned metabolic syndrome as the dependent variable and related indexes, such as WC, blood pressure, smoking and education as independent variables. The results show that except for smoking and blood sugar, other indicators were associated with metabolic syndrome $(\mathrm{p}<0.05)$ (Table 3$)$.

\subsection{ROC Curves of Indicators for Metabolic Syndrome}

ROC curves of the eight indicators with metabolic syndrome were calculated (Figure 1). The results show that in addition to the curve for FBS, other curves were located on top of the baseline $(p<0.05)$. The areas below the curves of BMI, TG, WC and TC were bigger than other areas. Therefore, BMI, TG, WC and TG are priority indicators for screening of metabolic syndrome (Table 4). As screening indicators of metabolic syndrome, the cut-off points for BMI was $24.78 \mathrm{~kg} / \mathrm{m}^{2}$ for males (sensitivity $=0.879$, specificity $=0.648$ ), and $24.72 \mathrm{~kg} / \mathrm{m}^{2}$ (sensitivity $=0.804$, specificity $=0.767$ ) for females. The cut-off point for WC was $85.95 \mathrm{~cm}$ (sensitivity $=$ 0.701 , specificity $=0.641$ ) for males and $78.25 \mathrm{~cm}$ (sensitivity $=0.804$, specificity $=0.528$ ) for females (Figure 2 and Figure 3).

\section{Discussion}

Univariate and multivariate analysis showed that BMI, WC, systolic blood pressure, diastolic blood pressure, blood glucose, total cholesterol, triglycerides and high density lipoprotein can be used as screening indicators of MS. The areas below the ROC curves of BMI, TG, WC and TC were bigger than those of other indicators. BMI, TG, WC and TC should be prior indicators for screening metabolic syndrome. TG and TC are biochemical indicators, and BMI and WC are physical indicators. However, measurement of BMI and WC are simple and rapid and therefore are more suitable for metabolic syndrome screening.

Table 2. Indicators of metabolic syndrome between case and control group ( $\bar{X} \pm \mathrm{S}$ ).

\begin{tabular}{ccccc}
\hline Indicators & Case group & Control group & $t$ & $\mathrm{p}$ \\
\hline BMI $\left(\mathrm{Kg} / \mathrm{m}^{2}\right)$ & $27.10 \pm 3.96$ & $23.31 \pm 3.92$ & -11.731 & $<0.01$ \\
WC (cm) & $88.02 \pm 10.18$ & $81.28 \pm 9.95$ & -8.202 & $<0.01$ \\
SBP (mmHg) & $139.80 \pm 23.23$ & $126.08 \pm 18.42$ & -8.01 & $<0.01$ \\
DBP (mmHg) & $86.87 \pm 12.97$ & $79.62 \pm 10.51$ & -7.52 & $<0.01$ \\
FBS (mmol/l) & $6.03 \pm 1.55$ & $5.49 \pm 0.58$ & -5.67 & $<0.01$ \\
TC (mmol/l) & $4.61 \pm 1.25$ & $3.82 \pm 1.17$ & -8.002 & $<0.01$ \\
TG (mmol/l) & $2.46 \pm 1.58$ & $1.40 \pm 1.02$ & -9.81 & $<0.01$ \\
HDLC (mmol/l) & $1.23 \pm 0.45$ & $1.10 \pm 0.37$ & -3.95 & 0.024 \\
\hline
\end{tabular}

Table 3. Multi-logistic analysis of metabolic syndrome.

\begin{tabular}{|c|c|c|c|c|c|c|}
\hline Indicator & B & S.E. & $X^{2}$ & $\mathrm{p}$ & OR & OR 95\% CI \\
\hline Smoking & -0.101 & 0.142 & 0.509 & 0.475 & 0.904 & $0.685-1.193$ \\
\hline Education & -0.169 & 0.059 & 8.120 & 0.004 & 0.844 & $0.751-0.949$ \\
\hline BMI $\left(\mathrm{kg} / \mathrm{m}^{2}\right)$ & 0.242 & 0.034 & 49.234 & 0.000 & 1.274 & $1.190-1.363$ \\
\hline $\mathrm{WC}(\mathrm{cm})$ & 0.087 & 0.022 & 15.093 & 0.000 & 1.091 & $1.044-1.140$ \\
\hline SBP (mmHg) & -0.064 & 0.022 & 8.381 & 0.004 & 0.938 & $0.899-0.980$ \\
\hline DBP (mmHg) & 0.029 & 0.008 & 11.437 & 0.001 & 1.029 & $1.012-1.046$ \\
\hline FBS (mmol/l) & 0.018 & 0.014 & 1.553 & 0.213 & 1.018 & $0.990-1.047$ \\
\hline $\mathrm{TC}(\mathrm{mmol} / \mathrm{l})$ & -1.376 & 0.244 & 31.875 & 0.000 & 0.253 & $0.157-0.407$ \\
\hline TG (mmol/l) & 1.076 & 0.152 & 50.200 & 0.000 & 2.934 & $2.179-3.952$ \\
\hline HDLC (mmol/l) & 1.961 & 0.389 & 25.403 & 0.000 & 7.104 & $3.314-15.227$ \\
\hline Constant & -18.894 & 1.977 & 91.287 & 0.000 & 0.000 & \\
\hline
\end{tabular}


Table 4. Areas under ROC curves for eight indicators used in metabolic syndrome screening.

\begin{tabular}{ccccc}
\hline Indicators & Areas & SE & p & $0.758-0.833$ \\
BMI & 0.796 & 0.019 & 0.000 & $0.651-0.735$ \\
WC & 0.693 & 0.022 & 0.000 & $0.639-0.725$ \\
SBP & 0.682 & 0.022 & 0.000 & $0.634-0.721$ \\
DBP & 0.677 & 0.022 & 0.000 & $0.576-0.671$ \\
FBS & 0.624 & 0.024 & 0.000 & $0.650-0.734$ \\
TC & 0.692 & 0.021 & 0.000 & $0.731-0.807$ \\
TG & 0.769 & 0.019 & 0.000 & $0.545-0.636$ \\
HDLC & 0.590 & 0.023 & 0.000 & \\
\hline
\end{tabular}

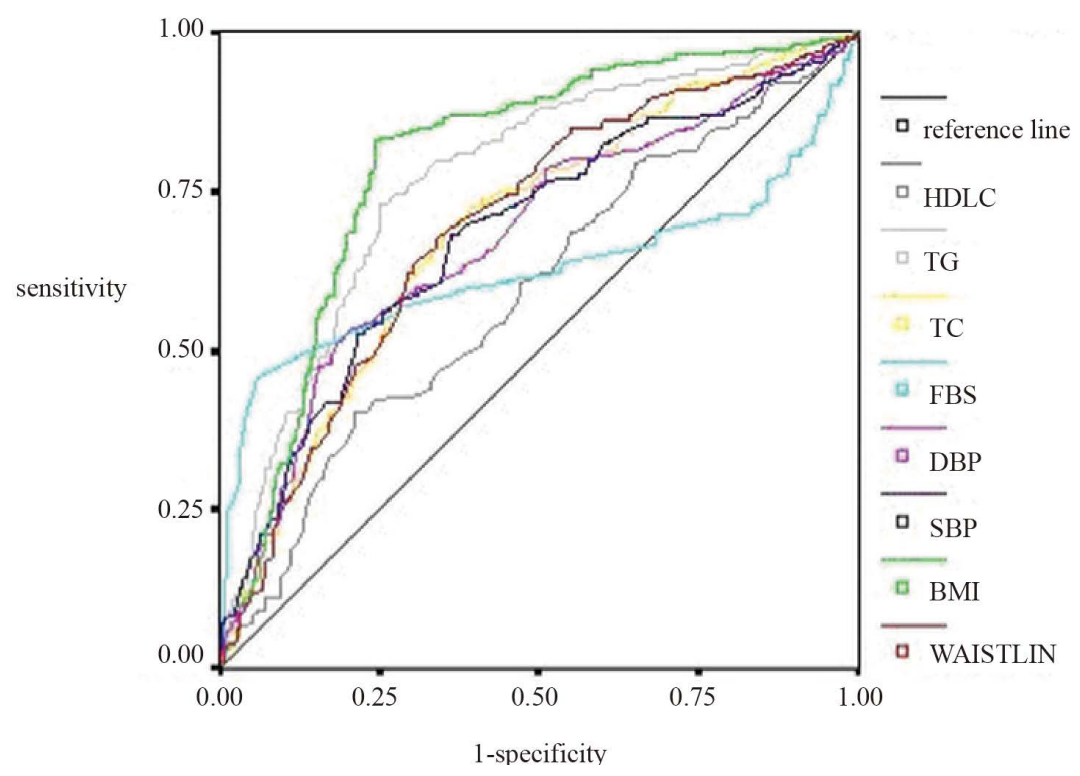

Figure 1. ROC curves of eight indicators for metabolic syndrome screening.

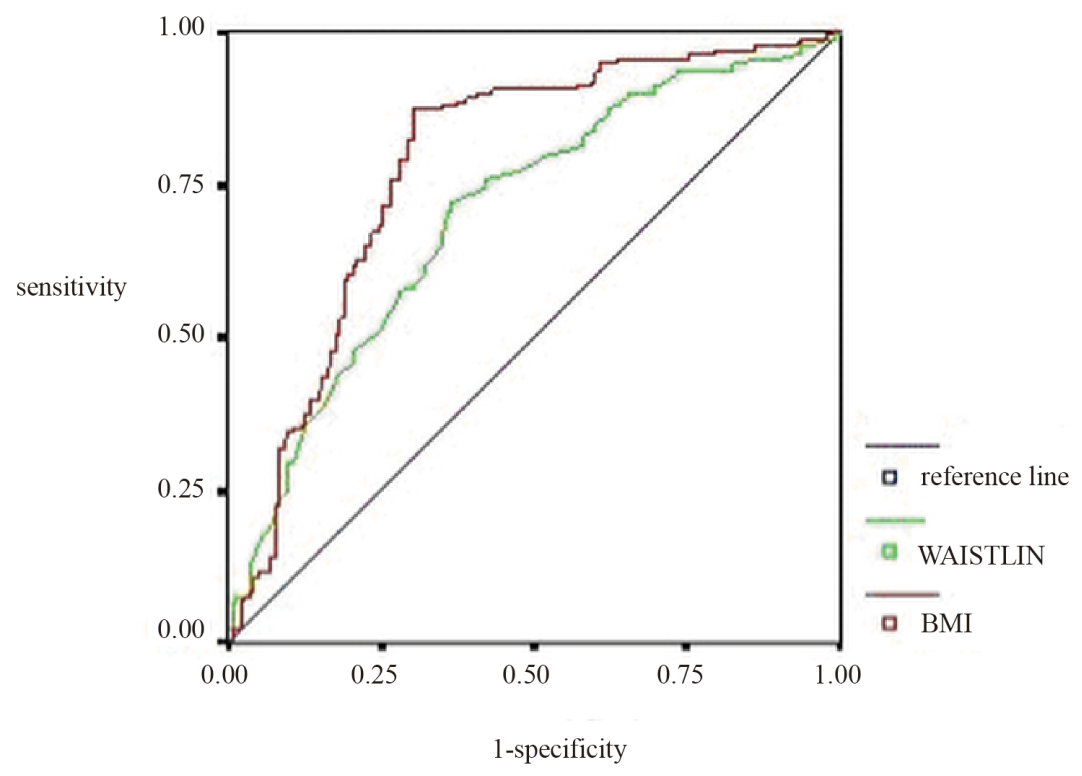

Figure 2. ROC curves of BMI and WC for metabolic syndrome screening of urban male residents in Ningxia. 


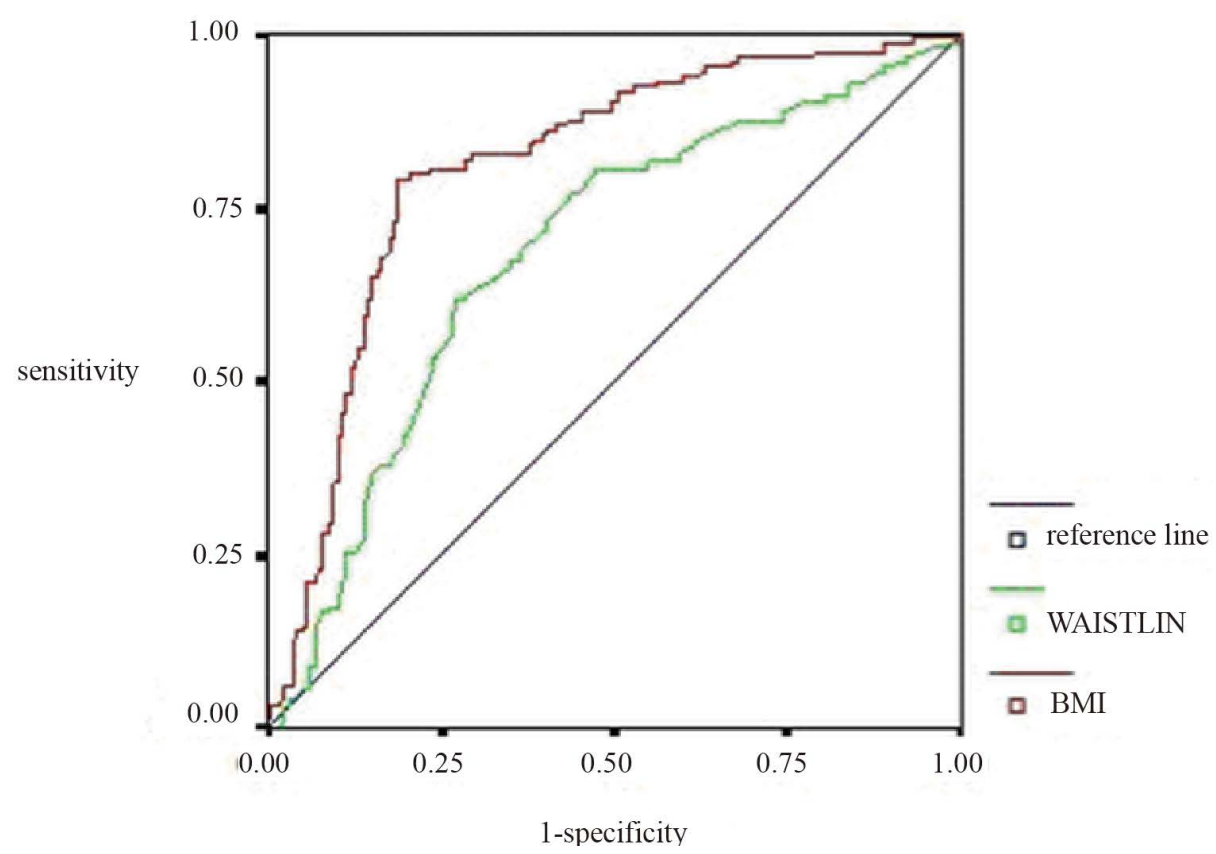

Figure 3. ROC curves of BMI and WC for metabolic syndrome screening of urban female residents in Ningxia.

Studies have confirmed that BMI and WC are closely linked with the occurrence and development of metabolic syndrome [7] [11]. BMI is the most widely used indicator of body fatness and been applied to both public health and clinical practice. In the context of national and international health care, BMI cut-offs are applied to population data to compare rates of overweight and obesity, develop policy, prevent programs, and assess the benefit of interventions. In a clinical context, BMI measurements are used to identify individuals at risk of co-morbidities, and in conjunction with other clinical variables to determine treatment regimes and monitor the effects of treatment [10].

The WHO recommends the use of pre-specified cut-off points for BMI and WC to standardize comparisons within and between populations [5]. Currently such cut-off points are derived from studies among European populations and thus may not be applicable to other ethnic groups [8] [14]. Indeed, some studies suggest that Asian populations manifest metabolic syndrome risk factors at lower levels of BMI and WC than Western populations [12] [13].

Based on sensitivity, specificity and ROC curve analysis, BMI values of $24.78 \mathrm{~kg} / \mathrm{m}^{2}$ (men) and $24.72 \mathrm{~kg} / \mathrm{m}^{2}$ (women) and WC values of $85.95 \mathrm{~cm}$ (men) and $78.25 \mathrm{~cm}$ (women) appear to best characterize high metabolic syndrome risk among people in Ningxia. These suggested cut-off points are consistent with other studies. For example, a BMI of $>23.2 \mathrm{~kg} / \mathrm{m}^{2}$ in Omani Arabs [15] [16], $\geq 25 \mathrm{~kg} / \mathrm{m}^{2}$ in Japan [9] and $27 \mathrm{~kg} / \mathrm{m}^{2}$ in Indonesia [17] was found to categorize obese individuals best in relation to high prevalent metabolic syndrome risk. Similarly, the reported cut-off points for WC range from $102 \mathrm{~cm}$ for American men [8], to $94 \mathrm{~cm}$ for Europeans [18], 84.5 $\mathrm{cm}$ for Omani Arabs [15], and $85 \mathrm{~cm}$ for Tunisians [16] and Japanese [9]. The corresponding figures among women are $88 \mathrm{~cm}$ for Americans [8], $80 \mathrm{~cm}$ for Europeans [10], Omani Arabs [15] and Asians [9], $85 \mathrm{~cm}$ for Tunisians [19] and $90 \mathrm{~cm}$ for Japanese [9]. For adults in China, the male WC cut-off points is $\geq 93 \mathrm{~cm}$ and female WC is $\geq 89 \mathrm{~cm}$ [14]. Last, a male WC of $\geq 85.0 \mathrm{~cm}$ and female $W C$ of $\geq 80.0 \mathrm{~cm}$ have been proposed as cut-off points of metabolic syndrome for identifying MS in the middle-aged and elderly in an urban community of Shanghai, China [11] [20].

Studies have shown that $60 \%$ of the global burden of metabolic syndrome occurs in developing countries, and it is the leading cause of disability in the world. As Ningxia is an ethnic minority region in China, it is important to increase the monitoring of WC for MS. Monitoring will benefit from the prevention and treatment of obesity and MS. The results of the present study will now be validated using a larger sample size, and the cut-off points for BMI and WC for Hui populations will be determined. However, our study has a few limitations. It relates the risk of MS to BMI and WC in a cross-sectional setting using the occurrence of established risk factors as a 
proxy risk estimate. This indicates the need for prospective studies that relate anthropometric measures to the incidence of diabetes, hypertension, dyslipidaemia and clinical MS.

\section{Conclusion}

We conclude that the two cut-off points for BMI and WC are lower in urban residents in Ningxia than the data currently recommended in Asian population. We encourage the use of these cut-off points when considering urban residents individuals in relation to their MS status. Prospective studies are needed to assess the incidence of diabetes, hypertension, dyslipidaemia and clinical MS.

\section{Acknowledgements}

This work was supported by the National Natural Science Foundation of China (No. 81060235). We thank doctors Wang Yancui of Guyuan city Maternal and Child Health Institute, and Shu Xuejun and Yang Yi of the centers for disease control and prevention for their administrative support during the performing stage of the study. We also thank Doctor Liu Xuewen of Shizuishan 905 community hospitals Ningxia, China for her assistance in laboratory analysis.

\section{References}

[1] Alberti, K.G.M., Zimmet, P. and Shaw, J., IDF Epidemiology Task Force Consensus Group (2005) The Metabolic Syndrome-A New Worldwide Definition. The Lancet, 366, 1059-1062. http://dx.doi.org/10.1016/S0140-6736(05)67402-8

[2] Chien, K.L., Hsu, H.C., Sung, F.C., Su, T.C., Chen, M.F. and Lee, Y.T. (2007) Metabolic Syndrome as a Risk Factor for Coronary Heart Disease and Stroke: An 11-Year Prospective Cohort in Taiwan Community. Atherosclerosis, 194, 214-221. http://dx.doi.org/10.1016/j.atherosclerosis.2006.07.033

[3] Bahrami, H., Sadatsafavi, M., Pourshams, A., Kamangar, F., Nouraei, M., Semnani, S. and Malekzadeh, R. (2006) Obesity and Hypertension in an Iranian Cohort Study; Iranian Women Experience Higher Rates of Obesity and Hypertension than American Women. BMC Public Health, 6, 158. http://dx.doi.org/10.1186/1471-2458-6-158

[4] Wiesenthal, S.R., Sandhu, H., McCall, R.H., Tchipashvili, V., Yoshii, H., Polonsky, K. and Giacca, A. (1999) Free Fatty Acids Impair Hepatic Insulin Extraction in Vivo. Diabetes, 48, 766-774. http://dx.doi.org/10.2337/diabetes.48.4.766

[5] World Health Organization (2000) Obesity: Preventing and Managing the Global Epidemic (No. 894). World Health Organization.

[6] Das, U.N. (2008) Essential Fatty Acids and Their Metabolites Could Function as Endogenous HMG-CoA Reductase and ACE Enzyme Inhibitors, Anti-Arrhythmic, Anti-Hypertensive, Anti-Atherosclerotic, Anti-Inflammatory, Cytoprotective, and Cardioprotective Molecules. Lipids in Health and Disease, 7, 18. http://dx.doi.org/10.1186/1476-511X-7-37

[7] Zhou, B.F., Wu, Y.F., Li, Y. and Zhang, L.F. (2005) The Cut-Off Point of Waist Circumference for Identifying Metabolic Syndrome in Chinese Adults. Chinese Journal of Cardiovascular Diseases, 33, 81-85.

[8] Saely, C.H., Koch, L., Schmid, F., Marte, T., Aczel, S., Langer, P. and Drexel, H. (2006) Adult Treatment Panel III 2001 but Not International Diabetes Federation 2005 Criteria of the Metabolic Syndrome Predict Clinical Cardiovascular Events in Subjects Who Underwent Coronary Angiography. Diabetes Care, 29, 901-907. http://dx.doi.org/10.2337/diacare.29.04.06.dc05-2011

[9] Kanazawa, M., Yoshiike, N., Osaka, T., Numba, Y., Zimmet, P. and Inoue, S. (2005) Criteria and Classification of Obesity in Japan and Asia-Oceania. World Review of Nutrition and Dietetics, 94, 1-12. http://dx.doi.org/10.1159/000088200

[10] Taylor, R.W., Brooking, L., Williams, S.M., Manning, P.J., Sutherland, W.H., Coppell, K.J. and Mann, J.I. (2010) Body Mass Index and Waist Circumference Cutoffs to Define Obesity in Indigenous New Zealanders. The American Journal of Clinical Nutrition, 92, 390-397. http://dx.doi.org/10.3945/ajcn.2010.29317

[11] Wildman, R.P., Gu, D., Reynolds, K., Duan, X. and He, J. (2004) Appropriate Body Mass Index and Waist Circumference Cutoffs for Categorization of Overweight and Central Adiposity among Chinese Adults. The American Journal of Clinical Nutrition, 80, 1129-1136.

[12] He, M., Tan, K.C., Li, E.T. and Kung, A.W. (2001) Body Fat Determination by Dual Energy X-Ray Absorptiometry and Its Relation to Body Mass Index and Waist Circumference in Hong Kong Chinese. International Journal of Obesity and Related Metabolic Disorders: Journal of the International Association for the Study of Obesity, 25, 748-752. 
http://dx.doi.org/10.1038/sj.ijo.0801612

[13] Chang, C.J., Wu, C.H., Chang, C.S., Yao, W.J., Yang, Y.C., Wu, J.S. and Lu, F.H. (2003) Low Body Mass Index but High Percent Body Fat in Taiwanese Subjects: Implications of Obesity Cutoffs. International Journal of Obesity, 27, 253-259. http://dx.doi.org/10.1038/sj.ijo.802197

[14] Zhou, B. (2002) Predictive Values of Body Mass Index and Waist Circumference to Risk Factors of Related Diseases in Chinese Adult Population. Chinese Journal of Epidemiology, 23, 5-10.

[15] Al-Lawati, J.A. and Jousilahti, P. (2008) Body Mass Index, Waist Circumference and Waist-to-Hip Ratio Cut-Off Points for Categorisation of Obesity among Omani Arabs. Public Health Nutrition, 11, 102-108. http://dx.doi.org/10.1017/S1368980007000183

[16] Ganguly, S.S., Al Shafaee, M.A., Al Lawati, J.A., Dutta, P.K. and Duttagupta, K.K. (2009) Epidemiological Transition of Some Diseases in Oman: A Situational Analysis. Eastern Mediterranean Health Journal, 15, 209-218.

[17] Gurrici, S., Hartriyanti, Y., Hautvast, J.G.A.J. and Deurenberg, P. (1998) Relationship between Body Fat and Body Mass Index: Differences between Indonesians and Dutch Caucasians. European Journal of Clinical Nutrition, 52, 779783. http://dx.doi.org/10.1038/sj.ejcn.1600637

[18] Alberti, K.G.M.M., Zimmet, P. and Shaw, J. (2006) Metabolic Syndrome-A New World-Wide Definition. A Consensus Statement from the International Diabetes Federation. Diabetic Medicine, 23, 469-480. http://dx.doi.org/10.1111/j.1464-5491.2006.01858.x

[19] Bouguerra, R., Alberti, H., Smida, H., Salem, L.B., Rayana, C.B., El Atti, J., Achour, A., Gaigi, S., Slama, C.B., Zouari, B. and Alberti, K.G. (2007) Waist Circumference Cut-Off Points for Identification of Abdominal Obesity among the Tunisian Adult Population. Diabetes, Obesity and Metabolism, 9, 859-868. http://dx.doi.org/10.1111/j.1463-1326.2006.00667.x

[20] Teng, X.Y., Liu, W., Jia, Y. and Fang, X.M. (2006) Optimum Variables and Corresponding Cut-Off Points for Identifying Obesity-Associated Metabolic Syndrome. Journal of Shanghai Jiao Tong University (Medical Science), 26, 980983. 